\title{
BMJ Open Relationship between Braden Scale scores and acute kidney injury among patients with acute coronary syndrome: a multicentre retrospective cohort study
}

\author{
Zhichao Li (D , , ${ }^{1,2}$ Bofu Liu, ${ }^{1}$ Dongze Li, ${ }^{1}$ Yu Jia, ${ }^{1}$ Lei Ye, ${ }^{1}$ Xiaoyang Liao, ${ }^{3}$ Zhi Zeng, ${ }^{1}$ \\ Zhi Wan ${ }^{1}$
}

To cite: Li Z, Liu B, Li D, et al. Relationship between Braden Scale scores and acute kidney injury among patients with acute coronary syndrome: a multicentre retrospective cohort study. BMJ Open 2022;12:e049102. doi:10.1136/ bmjopen-2021-049102

\section{- Prepublication history and} additional supplemental material for this paper are available online. To view these files, please visit the journal online (http://dx.doi.org/10.1136/ bmjopen-2021-049102).

Received 19 January 2021 Accepted 04 September 2021

Check for updates

(c) Author(s) (or their employer(s)) 2022. Re-use permitted under CC BY-NC. No commercial re-use. See rights and permissions. Published by BMJ.

${ }^{1}$ Department of Emergency Medicine, West China School of Nursing, West China Hospital, Sichuan University, Chengdu, China

${ }^{2}$ Department of Cardiology, West China Hospital, Sichuan University, Chengdu, China

${ }^{3}$ Department of General Practice and National Clinical Research Center for Geriatrics, West China Hospital, Sichuan University, Chengdu, China

Correspondence to Professor Zhi Wan; 303680215@qq.com and

Professor Zhi Zeng;

zengzhi2013@qq.com

\section{ABSTRACT}

Objectives To evaluate the impact of pressure ulcer events assessed by the Braden Scale (BS) on acute kidney injury (AKI) in patients with acute coronary syndrome (ACS).

Design A multicentre retrospective cohort study. Setting Chest pain centres from seven tertiary hospitals in China.

Participants We analysed 3185 patients with ACS from the Retrospective Evaluation of Acute Chest Pain study. The patients were divided into three groups (B1, B2 and B3) according to their BS scores ( $\leq 12$ vs $13-14$ vs $\geq 15$, respectively) at admission.

Outcome measures AKI was defined according to the criteria of the 2012 Kidney Disease: Improving Global Outcomes. Multivariate logistic analysis was used to evaluate the relationship between the BS score and AKI. Results There were 461 patients (14.5\%) with ACS who had the complication of AKI. Patients with a lower score on the BS had a higher incidence of AKI $(p<0.001)$. Multivariate logistic regression analysis showed that adjusted ORs of the BS score for AKI were 2.242 (B1 vs B3: $95 \% \mathrm{Cl}: 1.643$ to $3.060, \mathrm{p}<0.001$ ) and 1.566 (B2 vs B3: $95 \% \mathrm{Cl}: 1.186$ to $2.069, \mathrm{p}=0.002)$. The receiver operating characteristic curve analysis showed that the area under the curve of the BS score was $0.719(95 \% \mathrm{Cl}: 0.702$ to 0.736 ; $p<0.001$ ) for AKI.

Conclusions The BS score was independently associated with AKI. It may be a useful tool to identify those who may benefit from further prediction and prevention of AKI in patients with ACS.

Trail registration number ChiCTR1900024657 (http:// www.chictr.org.cn/). The satge rekates to results.

\section{INTRODUCTION}

Acute coronary syndrome (ACS), the acute manifestation of ischaemic heart disease, remains a major cause of morbidity and mortality worldwide ${ }^{1}$ and is responsible for more than 1 million hospital admissions in the USA annually. ${ }^{2}$ Although the mortality of patients with ACS has declined in recent years, the in-hospital mortality rate remains at $2.5 \%-4.2 \% .^{3-5}$ The identification of high-risk

\section{STRENGTHS AND LIMITATIONS OF THIS STUDY}

$\Rightarrow$ This is the first study to investigate the relationship between Braden Scale (BS) scores and acute kidney injury (AKI) in patients with acute coronary syndrome (ACS).

$\Rightarrow$ As a routine nursing evaluation programme, the BS tool should be considered by health professionals to stratify the risk of AKI for patients with ACS.

$\Rightarrow$ The causality between BS scores and AKI needs to be questioned because some patients already developed AKI before admission.

$\Rightarrow$ The creatinine level at first admission was set as the baseline creatinine level, which may reduce the detection rate of $\mathrm{AKI}$.

patients with ACS is important and facilitates therapeutic decision-making. ${ }^{6}$

Acute kidney injury (AKI) is a common complication of ACS that may be due to haemodynamic impairment and the use of contrast agents. ${ }^{7}$ According to a previous study, the incidence of AKI in patients with ACS ranges from $6.3 \%$ to $36.6 \%,{ }^{8}$ and AKI has a detrimental effect on the prognosis of patients, including prolonged length of hospital stay, higher incidence of cardiovascular events and twofold-threefold mortality. ${ }^{9-11}$ In view of the high incidence of AKI and its negative influence on the prognosis of patients with ACS, it is crucial to identify high-risk patients who may develop AKI so that medical staff can predict and prevent the occurrence of AKI in patients with ACS.

Frailty is a clinical syndrome, which results in the decline of body recovery, reserve capacity and resistance to stress. ${ }^{12}$ The overall frailty status of patients reportedly correlated with renal functional reserve $\mathrm{e}^{13-15}$ and was significantly associated with a high incidence of AKI. ${ }^{16}$ Therefore, early assessment and screening of frailty are critical to the assessment of AKI. 
The Braden Scale (BS) is widely used in routine nursing evaluation programmes to predict pressure ulcer events in patients. ${ }^{17}$ Because the BS examines several factors that could contribute to assessing frailty, such as nutrition, cognition, activity and function, it was recommended as a frailty identification tool. ${ }^{18}$ In our previous study, the low BS score was reportedly associated with death in patients with acute myocardial infection ${ }^{19}$; however, the relationship between the BS scores and AKI remains unclear. Clarifying the relationship may help nurses in daily nursing services identify patients with a high risk of AKI, avoid the use of nephrotoxic drugs, limit the dosage of contrast medium and make rational allocations of nursing management for high-risk patients with AKI. ${ }^{20} 21$ Therefore, this study aimed to evaluate the relationship between the BS score and AKI in patients with ACS undergoing primary percutaneous coronary intervention (PCI).

\section{MATERIALS AND METHODS \\ Study design}

In this multicentre retrospective cohort study, data from the Retrospective Evaluation of Acute Chest Pain (REACP) study were analysed to evaluate whether the BS could predict AKI in patients with ACS undergoing primary PCI. The REACP study enrolled patients with acute chest pain at chest pain centres from seven tertiary hospitals in China and registered them at www. chictr.org.cn (identifier: ChiCTR1900024657).

\section{Study population}

From January 2017 to February 2019, 14460 patients visited the acute chest pain centre, and 3337 adult patients were diagnosed with ACS and underwent primary PCI. Of these patients, 122 patients did not have relevant data and failed to return for follow-up, 150 patients were treated with thrombolysis and 218 patients left the hospital within 24 hours. Finally, 2847 patients were selected and divided into B1, B2 and B3

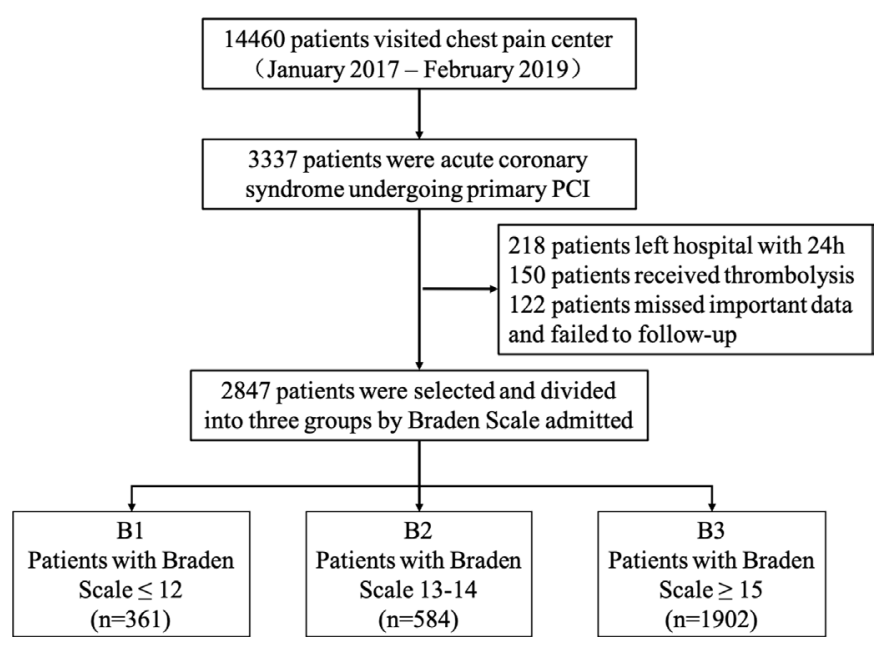

Figure 1 Study flow chart. $\mathrm{PCl}$, percutaneous coronary intervention. groups, according to the sum of their BS scores: $\leq 12$, 13-14 and $\geq 15$ on admission, respectively (figure 1 ).

\section{Data collection and definition}

We obtained data from the database of the REACP study. Data on vital signs, medical history, coronary angiography, medications, laboratory examination and imaging findings were collected by the physicians. Bedside echocardiography was performed by a professional technician within 24 hours after admission, and the left ventricular ejection fraction (LVEF) was calculated using the biplane Simpson method (Philips E33 Medical Systems, Bothell, Washington, USA). The Global Registry of Acute Coronary Events (GRACE) score and Gensini score were calculated according to previous studies. ${ }^{22-25}$ The Mehran risk score includes hypotension, use of intra-aortic balloon pump, congestive heart failure, age, anaemia, diabetes mellitus, contrast media volume and estimated glomerular filtration rate (eGFR) ${ }^{26}$ All data were reviewed by two trained clinical research coordinators.

At the emergency room, the protocol for the nurse is to use the BS on each patient at admission with re-evaluation every 24 hours. Six subscales constitute the BS, which includes sensation, communication, water, activity, nutrition and friction, and shear force. Each subscale has 1-4 points, excluding friction and shear force that have 1-3 points. Based on this scoring system, the total points can range from 6 to $23 .{ }^{17}$ According to the scientific interpretation of the BS in 2005, to identify the patient's risk of developing pressure ulcers, the scores were as follows: $15-23$, mild or no risk; $13-14$, moderate risk; and $\leq 12$, high risk. ${ }^{27}$

Staging of AKI was based on the criterion of Kidney Disease: Improving Global Outcomes. ${ }^{28}$ AKI stage 1 criteria are serum creatinine level $>26.5 \mathrm{mmol} / \mathrm{L}$ $(0.3 \mathrm{mg} / \mathrm{dL})$ within 48 hours, an increase in serum creatinine to 1.5-fold-1.9-fold of the baseline value, or urine output $<0.5 \mathrm{~mL} / \mathrm{kg} /$ hour for $6-12$ hours. The criteria for AKI stage 2 are an increase in serum creatinine to 2.0-fold-2.9-fold of the baseline value or urine output $<0.5 \mathrm{~mL} / \mathrm{kg} /$ hour for 12 hours. AKI stage 3 criteria are serum creatinine level $>353.6 \mathrm{mmol} / \mathrm{L}$ $(4.0 \mathrm{mg} / \mathrm{dL})$, an increase in serum creatinine more than 3.0 -fold of the baseline value, urine output $<0.3 \mathrm{~mL} /$ $\mathrm{kg}$ /hour for 24 hours or anuria for 12 hours. The first serum creatinine value measured on admission is considered the baseline serum creatinine.

\section{Endpoint and follow-up}

The study population underwent a median of 11.9 months (5.0-20.4 months) of follow-up. Trained physicians interviewed patients using structured telephone questionnaires. The primary endpoint was AKI.

\section{Statistical analysis}

The categorical variables were presented as numbers (percentages) and compared by the $\chi^{2}$ test. The 
Table 1 Demographics, triage vital signs, comorbidities and laboratory findings of patients with ACS in different BS group

\begin{tabular}{|c|c|c|c|c|}
\hline Variables & B1 $(n=361)$ & $B 2(n=584)$ & B3 $(n=1902)$ & $P$ value \\
\hline Age, years & $73.97 \pm 11.91$ & $72.25 \pm 11.23$ & $62.96 \pm 12.55$ & $<0.001$ \\
\hline Males, n (\%) & $120(33.2)$ & $202(34.6)$ & $374(19.7)$ & $<0.001$ \\
\hline Hypertension, n (\%) & $216(59.8)$ & $332(56.8)$ & $996(52.4)$ & 0.012 \\
\hline Diabetes, n (\%) & $137(38)$ & $180(30.8)$ & $480(25.2)$ & $<0.001$ \\
\hline COPD, n (\%) & $24(6.6)$ & $29(5)$ & $48(2.5)$ & $<0.001$ \\
\hline SBP, mm Hg & $123.83 \pm 28.25$ & $127.68 \pm 25.87$ & $128.49 \pm 23.15$ & 0.004 \\
\hline DBP, mm Hg & $73.41 \pm 18.22$ & $75.84 \pm 16.24$ & $79.12 \pm 15.16$ & $<0.001$ \\
\hline Heart rate, beats/min & $86.68 \pm 22.62$ & $83.34 \pm 19.38$ & $79.24 \pm 17.07$ & $<0.001$ \\
\hline Killip class $>1, \mathrm{n}(\%)$ & $244(67.6)$ & $319(54.6)$ & $725(38.1)$ & $<0.001$ \\
\hline \multicolumn{5}{|l|}{ Laboratory findings } \\
\hline WBC, $10^{9} / \mathrm{L}$ & $9.67(7.3-12.31)$ & $9.2(6.93-12.32)$ & $8.86(6.91-11.29)$ & 0.001 \\
\hline Platelet count, $10^{9} / \mathrm{L}$ & $184.62 \pm 77.08$ & $187.76 \pm 80.5$ & $183.9 \pm 72.02$ & 0.551 \\
\hline Blood glucose, $\mathrm{mmol} / \mathrm{L}$ & $10.43 \pm 5.33$ & $9.24 \pm 4.46$ & $8.53 \pm 3.95$ & $<0.001$ \\
\hline Creatinine, $\mu \mathrm{mol} / \mathrm{L}$ & 97 (75.75-145.25) & $83(69-110)$ & 77 (65-92) & $<0.001$ \\
\hline eGFR & $60.12 \pm 28.14$ & $67.87 \pm 26.25$ & $83.07 \pm 23.49$ & $<0.001$ \\
\hline $\mathrm{BUN}, \mathrm{mmol} / \mathrm{L}$ & $9.58 \pm 6.89$ & $7.92 \pm 4.96$ & $6.29 \pm 3.42$ & $<0.001$ \\
\hline Triglycerides, $\mathrm{mmol} / \mathrm{L}$ & $1.19(0.84-1.62)$ & $1.19(0.84-1.73)$ & $1.41(0.97-2.12)$ & $<0.001$ \\
\hline Total cholesterol, $\mathrm{mmol} / \mathrm{L}$ & $4.11 \pm 1.31$ & $4.3 \pm 1.15$ & $4.48 \pm 1.26$ & $<0.001$ \\
\hline Contrast dose, $\mathrm{mL}$ & $131.88 \pm 30.33$ & $125.21 \pm 27.03$ & $120.04 \pm 23.16$ & $<0.001$ \\
\hline Stent & $1(1-2)$ & $1(1-2)$ & $1(1-2)$ & 0.208 \\
\hline Three-vessel disease, $n(\%)$ & $100(27.7)$ & $117(20.0)$ & $298(15.7)$ & $<0.001$ \\
\hline Procedure time, $\min$ & $41.92 \pm 10.66$ & $41.14 \pm 10.25$ & $39.85 \pm 9.85$ & $<0.001$ \\
\hline \multicolumn{5}{|l|}{ Risk score } \\
\hline GRACE score & $178.55 \pm 43.95$ & $163.39 \pm 38.6$ & $137.43 \pm 35.12$ & $<0.001$ \\
\hline Gensini score & $80(34-124)$ & 66 (37-103) & $57(30-90)$ & $<0.001$ \\
\hline Mehran risk score & $15.47 \pm 5.15$ & $13.85 \pm 5.15$ & $11.17 \pm 5.01$ & $<0.001$ \\
\hline
\end{tabular}

ACS, acute coronary syndrome; BMI, body mass index; BS, Braden Scale; BUN, blood urea nitrogen; COPD, chronic obstructive pulmonary disease; CTnT, cardiac troponin T; DBP, diastolic blood pressure; eGFR, estimated glomerular filtration rate; GRACE, Global Registry of Acute Coronary Events; HDL, high-density lipoprotein; LDL, low-density lipoprotein; LVEF, left ventricular ejection fraction; NT-proBNP, N-terminal pro-brain natriuretic peptide; $\mathrm{PCI}$, percutaneous coronary intervention; SBP, systolic blood pressure; STEMI, ST-segment elevation myocardial infarction; WBC, white blood cell count.

continuous variables were reported as medians (25th75 th) or means \pm SDs, according to non-normal and normal distribution and compared using the MannWhitney $\mathrm{U}$ test or analysis of variance, respectively.
The logistic regression model was used to evaluate whether BS scores are associated with AKI. All variables were included in a univariate model, and the significant variables were re-entered into a multivariable model. The area under the receiver operating 

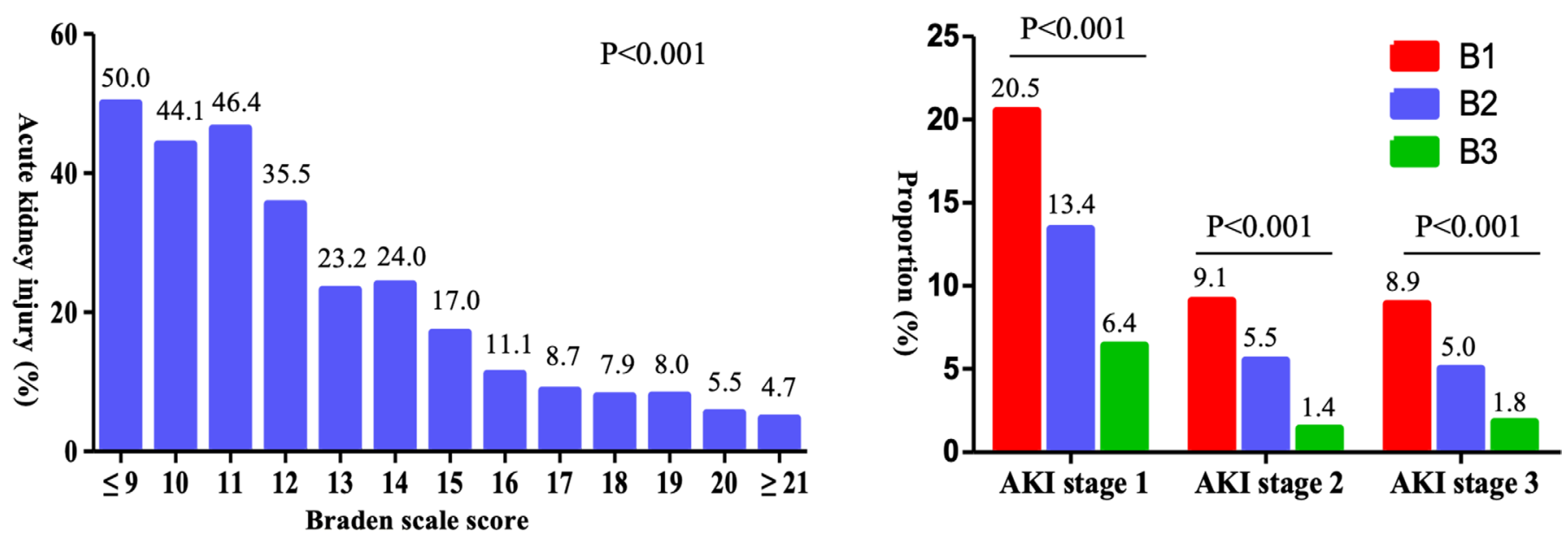

Figure 2 The incidence of AKI in patients with different BS score. AKI, acute kidney injury; BS, Braden Scale.

characteristic (ROC) curves was established to evaluate the ability of the BS scores to predict AKI. The cumulative survival rates among the three groups of patients (B1 vs B2 vs B3) with or without AKI were compared by the Kaplan-Meier curve. To explore the indirect effect of the BS scores on all-cause mortality through the bypath of AKI, path analysis established by structural equation modelling was performed. ${ }^{29}$ The results of the path analysis were analysed by standardised regression coefficients $(\beta)$ to describe the direct and indirect effects on all-cause mortality, and the proportion of the mediating effect was calculated by dividing the regression coefficient of the indirect path by the total regression coefficient. A two-tailed $\mathrm{p}$ value of $<0.05$ was considered significant. Data were analysed using SPSS Statistics V.20.0 and R for Windows V.3.5.0.

\section{Patient and public involvement}

The patients were not involved in the design of or data collection in this study.

\section{RESULTS}

\section{Baseline patient characteristics}

The mean age of the 2847 patients with ACS was $66.26 \pm 13.08$ years, and $2151(75.5 \%)$ of them were men. There were 361 patients (12.7\%), 584 patients $(20.5 \%)$ and 1902 patients $(66.8 \%)$ assigned to B1, B2 and B3 groups, respectively. Baseline characteristics of the patients in the B1 group were that they were older and more likely to have complications, such as hypertension, diabetes and Killip class $\geq \mathrm{II}$, and have higher baseline renal function indicators, cardiac biomarkers, consumption of contrast agent, GRACE score, Gensini score and Mehran risk score $(\mathrm{p}<0.05$, table 1$)$. The baseline characteristics of patients divided into non-AKI, AKI stage 1, AKI stage 2 and AKI stage 3 are compared in online supplemental table 1.

\section{The relationship between the BS and AKI}

With the increase in the BS scores, the incidence of AKI gradually decreased $(p<0.001)$. Patients with lower BS

Table 2 Univariate and multivariate logistic regression analysis of Braden Scale for acute kidney injury

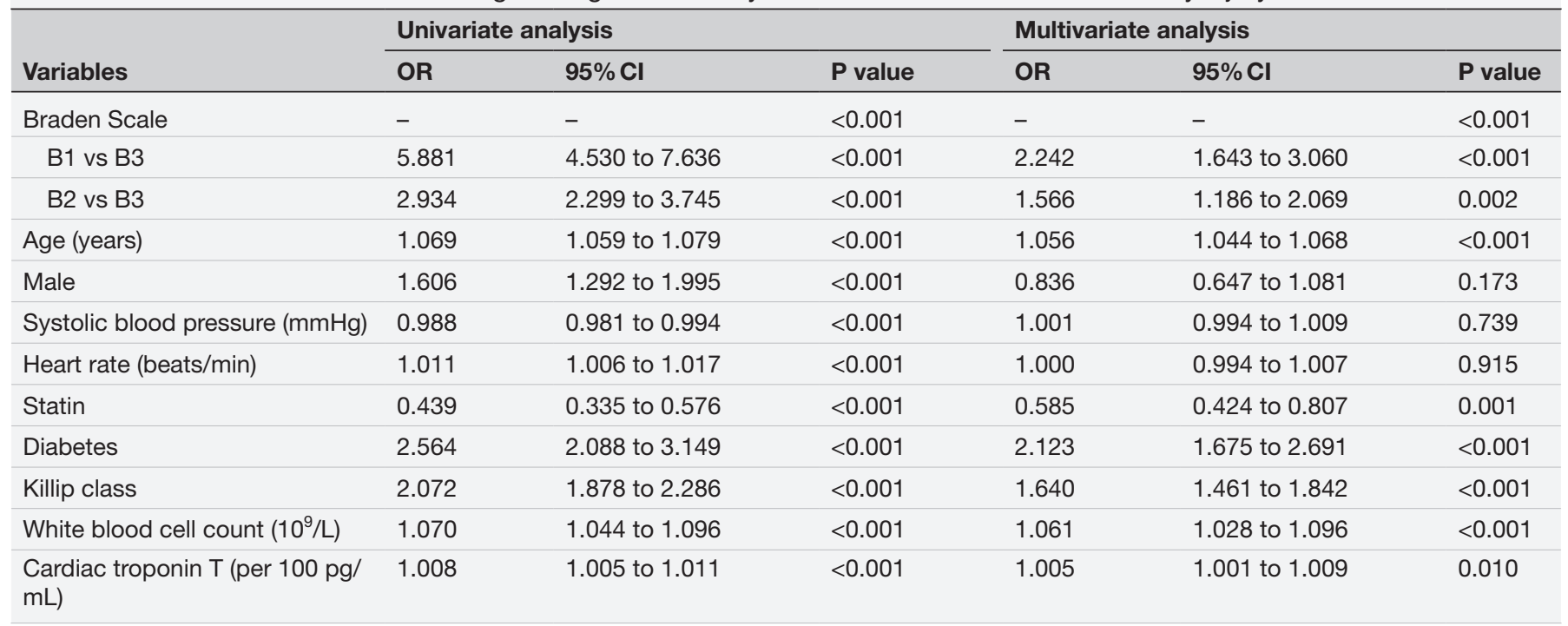




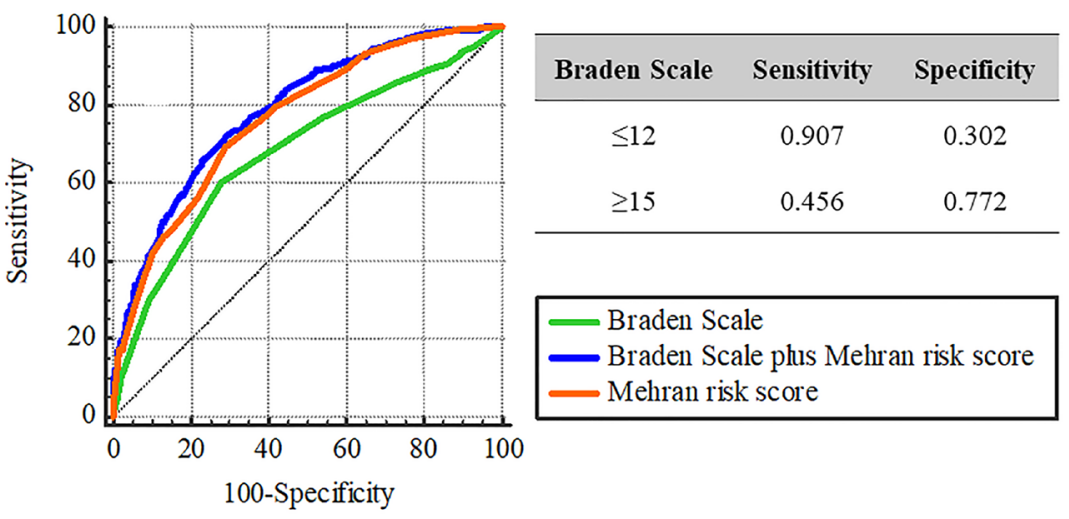

\begin{tabular}{ccccc}
\hline Predictors & AUC & $\mathbf{9 5 \% \text { CI }}$ & P for AUC & $\begin{array}{c}\text { P for AUC } \\
\text { comparison }\end{array}$ \\
\hline Braden Scale & 0.681 & $0.653-0.71$ & $<0.0001$ & $<0.0001$ \\
Mehran risk score & 0.765 & $0.742-0.788$ & $<0.0001$ & Reference \\
Combined & 0.785 & $0.763-0.807$ & $<0.0001$ & 0.0001 \\
\hline
\end{tabular}

Figure 3 ROC curve analysis according to BS score for AKI in patients with ACS. ACS, acute coronary syndrome; AKI, acute kidney injury; AUC, area under the curve; BS, Braden Scale; ROC, receiver operating characteristic.

scores had a higher incidence of AKI $(38.5 \%$ vs $23.9 \%$ vs $9.6 \%)$ and AKI stage 1 (20.5\% vs $13.4 \%$ vs $6.4 \%)$, AKI stage $2(9.1 \%$ vs $5.5 \%$ vs $1.4 \%)$ and AKI stage $3(8.9 \%$ vs $5.0 \%$ vs $1.8 \%$ ) (figure 2).

In univariate logistic regression analysis, BS score, age, male sex, systolic blood pressure, heart rate, statin level, diabetes, Killip class, white blood cell count and cardiac troponin $\mathrm{T}$ level were significantly related with AKI. After adjusting all significant factors, the multivariate logistic regression model showed that decreased BS scores were independent predictors for AKI (B1 vs B3: OR: 2.242, 95\% CI: 1.643 to 3.060, $\mathrm{p}=0.002$; B2 vs B3: OR: $1.566,95 \%$ CI: 1.186 to $2.069, \mathrm{p}<0.001$, table 2 ).

In the ROC curve analysis, the BS scores showed a significant prediction efficiency for AKI (area under the curve (AUC): $0.719 ; 95 \%$ CI: 0.702 to $0.736 ; \mathrm{p}<0.001$, figure 3 ). The cut-off value of BS scores was set at 12 , yielding a high sensitivity of $90.7 \%$ and a specificity of $30.2 \%$ for AKI. Moreover, when the cut-off value of BS scores was set at 15 , the sensitivity and specificity for AKI were $45.6 \%$ and $77.2 \%$, respectively. Moreover, BS scores improved the AUC of Mehran risk score from 0.765 (95\% CI: 0.742 to 0.788 ) to 0.785 (95\% CI: 0.763 to 0.807 ), which was significantly greater than that of Mehran risk score alone $(\mathrm{p}<0.001)$.

\section{Subgroup analysis}

The OR for AKI was calculated after grouping the patients by age, sex, systolic blood pressure, heart rate, statin level, diabetes, Killip class, white blood cell count and cardiac troponin T. A lower BS score was still associated with a higher incidence of AKI in patients with ACS (table 3).

\section{The interaction among BS groups, AKI and mortality}

Overall, 126 patients $(4.4 \%)$ died in the hospital, and $345(12.1 \%)$ total deaths were recorded during the follow-up period. Patients with lower BS or AKI have higher all-cause mortality and longer length of stay $(p<0.001)$ in the hospital and higher all-cause mortality during the follow-up period $(\mathrm{p}<0.001$, online supplemental tables 2 and 3).

Kaplan-Meier analysis showed that the cumulative survival rate of patients with lower BS scores or AKI was lower than that of patients without these factors in patients with ACS (B1+AKI vs B2+AKI vs B1+non-AKI vs B3+AKI vs B2+non-AKI vs B3+non-AKI: $35.2 \%$ vs $60.4 \%$ vs $67.4 \%$ vs $77.9 \%$ vs $81.0 \%$ vs $92.5 \%$, figure 4 ).

The BS score was significantly associated with both AKI and all-cause mortality during the follow-up period according to path analysis $(p<0.001)$. In the structural equation model, the indirect impact of the BS score on long-term all-cause mortality mediated by AKI accounted for $30 \%$ (figure 5 ).

\section{DISCUSSION}

Our study found that the incidence of AKI gradually increased with the decrease of BS scores in patients with ACS. According to multivariate logistic regression analysis, BS scores were independently related to AKI in patients with ACS. In subgroup analysis, this association 
Table 3 Logistic regression analysis of AKI in different subgroups according to BS

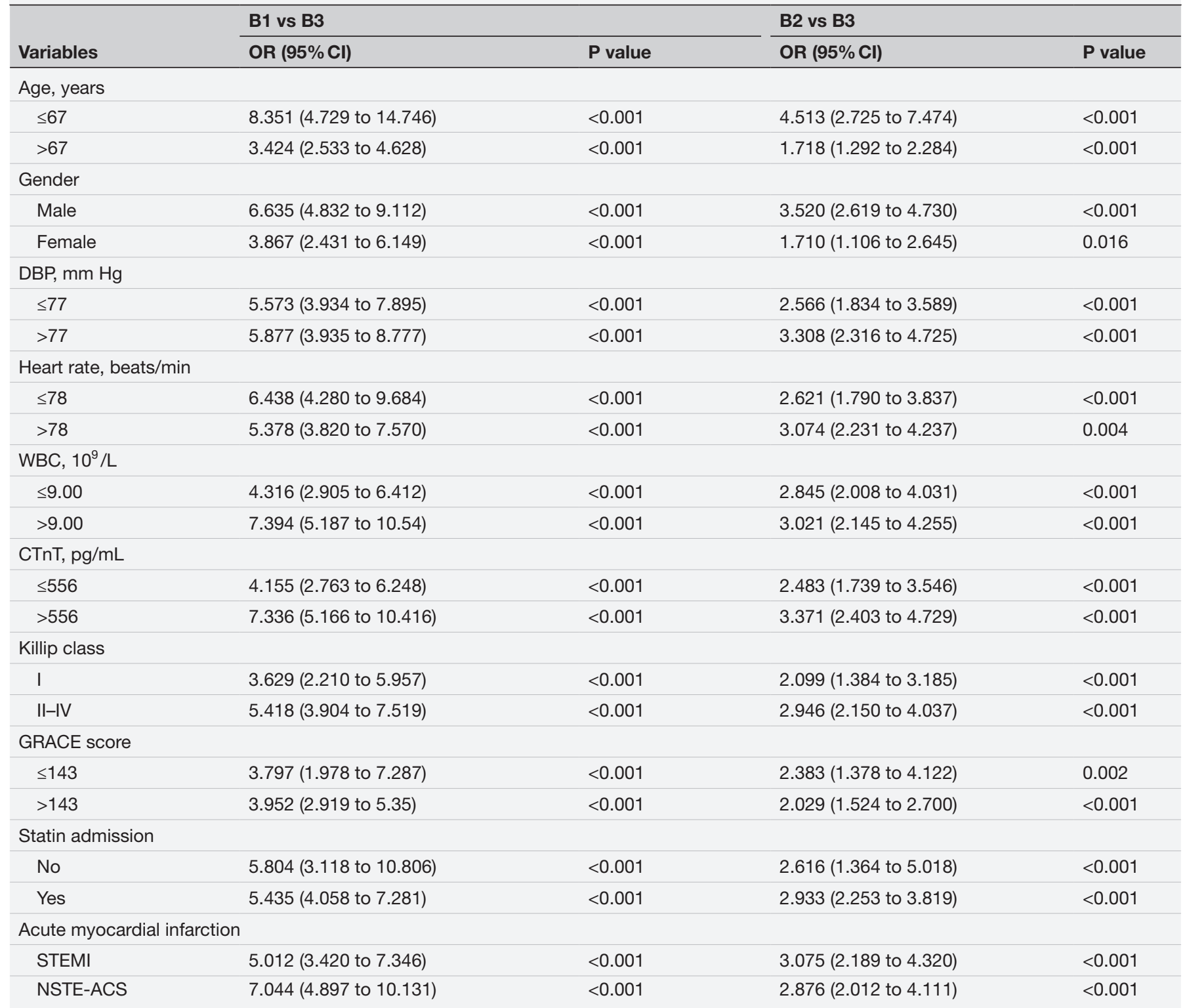

The continuous variables were grouped by median.

AKI, acute kidney injury; BS, Braden Scale; CTnT, cardiac troponin T; DBP, diastolic blood pressure; GRACE, Global Registry of Acute Coronary Events; NSTE-ACS, non-ST-segment elevated acute coronary syndrome; STEMI, ST-segment elevated myocardial infarction; WBC, white blood cell count.

was consistent in patients with different characteristics. Furthermore, the BS score achieved a significant incremental predictive value beyond the Mehran risk scores, which is a widely validated contrast-induced nephropathy assessment index. ${ }^{30} 31$ Therefore, in clinical nursing practice, the BS cannot only be used to assess the risk of pressure ulcer ${ }^{19}{ }^{32}$ but can also be used as a tool for the risk assessment of AKI.

According to a meta-analysis, the incidence of AKI in patients with ACS was as high as $15.8 \%$, which was similar to our findings in this study, and the incidence of AKI in the present study was $16.2 \%{ }^{8}$ Considering that numerous studies have confirmed the adverse effects of AKI on the prognosis of patients with ACS, predicting the incidence of AKI is a key measure for early risk stratification of patients with ACS.

As in previous studies, both BS scores and AKI were risk factors for death ${ }^{10}{ }^{19}$; therefore, we aimed to further clarify the interaction between BS scores, AKI and death. The Kaplan-Meier survival analysis was constructed, and the results showed that the cumulative survival rate of ACS patients with lower BS scores and/or AKI was lower than that of patients with ACS without these factors. More importantly, the path analysis by the structural equation model showed that the approximately $30 \%$ indirect impact of BS scores on long-term all-cause mortality was mediated by AKI. It was suggested that BS scores were 


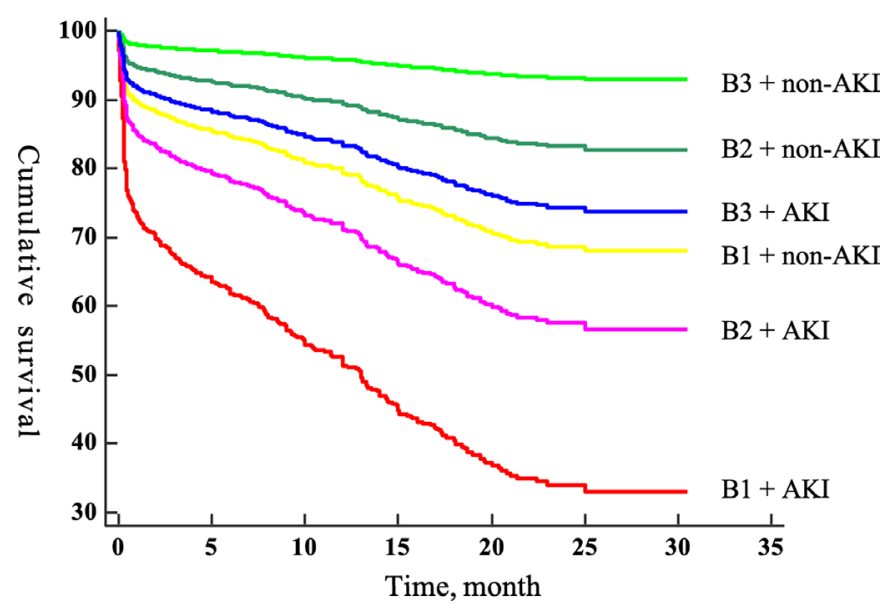

Figure 4 Kaplan-Meier survival curve of long-term all-cause mortality in different BS score patients with or without AKI. AKI, acute kidney injury; BS, Braden Scale.

closely related to AKI, and their interaction increases the occurrence of mortality events.

Although the BS is a widely used tool for the routine nursing assessment of pressure ulcer events, it evaluates several indicators related to frailty, such as nutrition, cognition and function. Therefore, recent studies recommended it as a tool to identify frailty. ${ }^{18}$ However, there may be other mechanisms to explain the relationship between BS scores and AKI, given that a low BS score does not necessarily indicate frailty. In fact, patients show lower cognition, function and activity in the acute stage of ischaemic events, which may be affected by the severity of the disease itself. Even though the BS score reflects the frailty from some aspects, the BS score on admission does not represent the BS score for patients under the usual state. As shown, patients with lower BS scores have higher Killip class and cardiac marker levels, lower LVEF and diastolic blood pressure, and higher incidence of cardiogenic shock, reflecting a more severe haemodynamic impairment. In addition, patients with lower BS

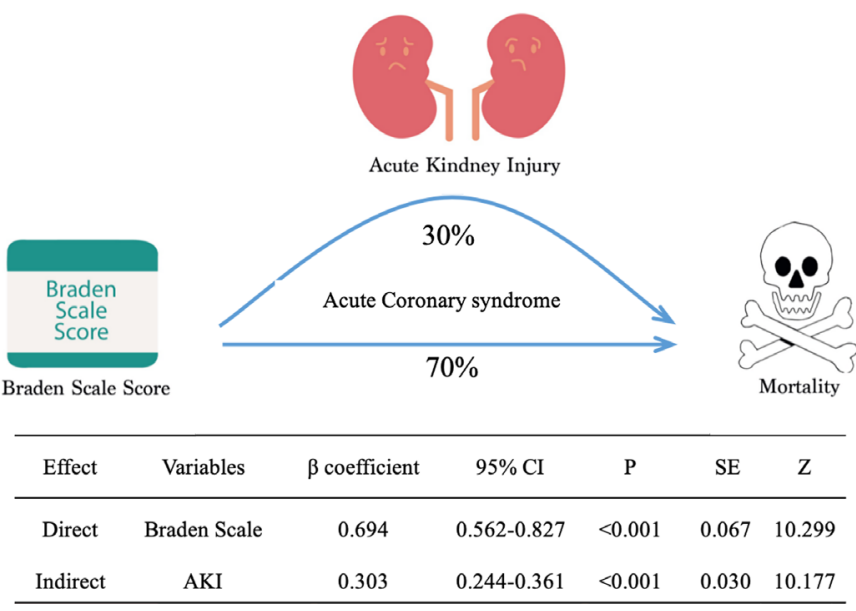

Figure 5 Direct and indirect effects of admission BS score on AKI and long-term all-cause mortality. $\beta$ coefficient was calculated by the standard regression equation. AKI, acute kidney injury; BS, Braden Scale. scores have higher frequency of multiple vessel lesions and more severe coronary stenosis, which may explain the increased consumption of contrast agents. Finally, higher baseline indicators for inflammation, thrombosis and stress may also be related to patients with lower BS scores. All of these may be potential contributors to AKI. Moreover, several studies have shown that the assessment of peripheral arterial disease is helpful to predict contrastinduced AKI. ${ }^{33}$ At the same time, peripheral artery disease has been proved to be an independent factor of pressure ulcer and related to BS scores. ${ }^{35-37}$ Given that the BS consists of activity, movement and perception, it may reflect the severity of peripheral arterial disease and indirectly assess the risk of AKI events. Therefore, the BS score prediction of AKI events may be due to several factors.

For patients at risk of AKI assessed by the BS score, nurses should administer nephrotoxic drugs cautiously, reduce the dose of contrast agents rationally, ensure adequate hydration and maintain renal perfusion. ${ }^{20} 21$ In addition to increasing the preventive measures for AKI, nurses should prevent the adverse impact of frailty on AKI based on comprehensive interventions aimed at improving the prognosis of patients with ACS and improving BS scores, such as adequate nutrition intake, provision of social support, maintenance of cognitive function and ability to perform daily activities, which may ameliorate the vicious circle between frailty and AKI. ${ }^{38} 39$

\section{CONCLUSIONS}

The BS score at admission was independently associated with AKI for patients with ACS. The BS may be a useful and simple tool to identify the risk of AKI among patients with ACS. In addition, more than $30 \%$ of the effects of the BS score on mortality were mediated by AKI, which suggests that frailty and AKI are two important intervention targets to improve the prognosis of patients with ACS.

Acknowledgements We are grateful for the support of the cooperating institutions participating in this research. We also sincerely thank the researchers for their contributions to this research.

Contributors ZL, DL, ZZ and ZW conceived and planned the study. ZL, BL and YJ performed the analysis. ZL, BL, ZZ and ZW wrote the original draft with input from all authors. ZL, BL and YJ visualised the results. ZL, BL, YJ and DL contributed to the interpretation of the results. $\mathrm{XL}, \mathrm{ZZ}$ and $\mathrm{ZW}$ did critical revision of the article. ZZ and ZW supervised the entire process. All the authors read and approved the final manuscript. All authors take responsibility for the integrity of the data and accuracy of the data analysis. ZL was responsible for the overall content as guarantor. ZL accepts full responsibility for the finished work and/or the conduct of the study, had access to the data, and controlled the decision to publish.

Funding This work was supported financially by grants from Sichuan Science and Technology Program (numbers: 2020YFS0154, 2020YFSY0014 and 2019JDRC0105), West China Hospital, Sichuan University (numbers: 2018HXFH001, 2018HXFH027 and 20HXFH050) and West China School of Nursing, Sichuan University (number: HXHL20046, HXHL19023).

Competing interests None declared.

Patient consent for publication Not applicable. 
Ethics approval The study complied with the Declaration of Helsinki, and the Human Ethical Committee of West China Hospital of Sichuan University approved the study protocol.

Provenance and peer review Not commissioned; externally peer reviewed.

Data availability statement Data are available upon reasonable request. Data are available upon reasonable request. The data are available upon request from the corresponding author (303680215@qq.com).

Supplemental material This content has been supplied by the author(s). It has not been vetted by BMJ Publishing Group Limited (BMJ) and may not have been peer-reviewed. Any opinions or recommendations discussed are solely those of the author(s) and are not endorsed by BMJ. BMJ disclaims all liability and responsibility arising from any reliance placed on the content. Where the content includes any translated material, BMJ does not warrant the accuracy and reliability of the translations (including but not limited to local regulations, clinical guidelines, terminology, drug names and drug dosages), and is not responsible for any error and/or omissions arising from translation and adaptation or otherwise.

Open access This is an open access article distributed in accordance with the Creative Commons Attribution Non Commercial (CC BY-NC 4.0) license, which permits others to distribute, remix, adapt, build upon this work non-commercially, and license their derivative works on different terms, provided the original work is properly cited, appropriate credit is given, any changes made indicated, and the use is non-commercial. See: http://creativecommons.org/licenses/by-nc/4.0/.

ORCID iD

Zhichao Li http://orcid.org/0000-0002-5105-3570

\section{REFERENCES}

1 Roth GA, Johnson C, Abajobir A, et al. Global, regional, and national burden of cardiovascular diseases for 10 causes, 1990 to 2015. J Am Coll Cardiol 2017;70:1-25.

2 Eisen A, Giugliano RP, Braunwald E. Updates on acute coronary syndrome: a review. JAMA Cardiol 2016;1:718-30.

3 Virani SS, Alonso A, Benjamin EJ, et al. Heart disease and stroke Statistics-2020 update: a report from the American heart association. Circulation 2020;141:e139-596.

4 Malanchini G, Stefanini GG, Malanchini M, et al. Higher in-hospital mortality during weekend admission for acute coronary syndrome: a large-scale cross-sectional Italian study. J Cardiovasc Med 2019;20:74-80.

5 Peng Y, Du X, Rogers KD, et al. Predicting in-hospital mortality in patients with acute coronary syndrome in China. Am J Cardiol 2017;120:1077-83.

6 Loudon BL, Gollop ND, Carter PR, et al. Impact of cardiovascular risk factors and disease on length of stay and mortality in patients with acute coronary syndromes. Int J Cardiol 2016;220:745-9.

7 Almendarez M, Gurm HS, Mariani J, et al. Procedural strategies to reduce the incidence of contrast-induced acute kidney injury during percutaneous coronary intervention. JACC Cardiovasc Interv 2019;12:1877-88.

8 Pickering JW, Blunt IRH, Than MP. Acute kidney injury and mortality prognosis in acute coronary syndrome patients: a meta-analysis. Nephrology 2018;23:237-46.

9 Kaltsas E, Chalikias G, Tziakas D. The incidence and the prognostic impact of acute kidney injury in acute myocardial infarction patients: current preventive strategies. Cardiovasc Drugs Ther 2018;32:81-98.

10 Marenzi G, Cosentino N, Bartorelli AL. Acute kidney injury in patients with acute coronary syndromes. Heart 2015;101:1778-85.

11 Marenzi G, Cabiati A, Bertoli SV, et al. Incidence and relevance of acute kidney injury in patients hospitalized with acute coronary syndromes. Am J Cardiol 2013;111:816-22.

12 Ortiz-Soriano V, Neyra JA. The impact of acute kidney injury on frailty status in critical illness survivors-is there enough evidence? J Emerg Crit Care Med 2018;2. doi:10.21037/jeccm.2018.10.17. [Epub ahead of print: 07112018 ]

13 Guerville F, de Souto Barreto P, Taton B, et al. Estimated glomerular filtration rate decline and incident frailty in older adults. Clin $\mathrm{J} \mathrm{Am}$ Soc Nephrol 2019;14:1597-604.

14 Morton S, Isted A, Avery P, et al. Is frailty a predictor of outcomes in elderly inpatients with acute kidney injury? A prospective cohort study. Am J Med 2018;131:1251-6.
15 Baek SH, Lee SW, Kim S-W, et al. Frailty as a predictor of acute kidney injury in hospitalized elderly patients: a single center, retrospective cohort study. PLoS One 2016;11:e0156444.

16 Jiesisibieke ZL, Tung T-H, Xu Q-Y, et al. Association of acute kidney injury with frailty in elderly population: a systematic review and metaanalysis. Ren Fail 2019;41:1021-7.

17 Brown SJ. The Braden scale. A review of the research evidence. Orthop Nurs 2004;23:30-8.

18 Cohen R-R, Lagoo-Deenadayalan SA, Heflin MT, et al. Exploring predictors of complication in older surgical patients: a deficit accumulation index and the Braden scale. J Am Geriatr Soc 2012;60:1609-15.

19 Jia Y, Li H, Li D, et al. Prognostic value of Braden scale in patients with acute myocardial infarction: from the retrospective multicenter study for early evaluation of acute chest pain. J Cardiovasc Nurs 2020;35:E53-61.

20 Kashani K, Rosner MH, Haase M, et al. Quality improvement goals for acute kidney injury. Clin J Am Soc Nephrol 2019;14:941-53.

21 Murphy F, Byrne $\mathrm{G}$. The role of the nurse in the management of acute kidney injury. Br J Nurs 2010;19:146-52.

22 Granger CB, Goldberg RJ, Dabbous O, et al. Predictors of hospital mortality in the global registry of acute coronary events. Arch Intern Med 2003;163:2345-53.

23 Gensini GG. A more meaningful scoring system for determining the severity of coronary heart disease. Am J Cardiol 1983;51:606.

24 Jia Y, Gao Y, Li D, et al. Geriatric nutritional risk index score predicts clinical outcome in patients with acute ST-segment elevation myocardial infarction. J Cardiovasc Nurs 2020;35:E44-52.

25 Yu J, Li D, Jia Y, et al. Nutritional risk screening 2002 was associated with acute kidney injury and mortality in patients with acute coronary syndrome: insight from the REACP study. Nutr Metab Cardiovasc Dis 2021;31:1121-8.

26 Mehran R, Aymong ED, Nikolsky E, et al. A simple risk score for prediction of contrast-induced nephropathy after percutaneous coronary intervention: development and initial validation. J Am Coll Cardiol 2004;44:1393-9.

27 Braden BJ, Maklebust J, Maklebust J. Preventing pressure ulcers with the Braden scale: an update on this easy-to-use tool that assesses a patient's risk. Am J Nurs 2005;105:70-2.

28 Khwaja A. KDIGO clinical practice guidelines for acute kidney injury. Nephron Clin Pract 2012;120:c179-84.

29 Stein CM, Morris NJ, Nock NL. Structural equation modeling. Methods Mol Biol 2012;850:495-512.

30 Koowattanatianchai S, Chantadansuwan T, Kaladee A, et al. Practical risk stratification score for prediction of contrast-induced nephropathy after primary percutaneous coronary intervention in patients with acute ST-segment elevation myocardial infarction. Cardiol Res 2019;10:350-7.

31 Abellás-Sequeiros RA, Raposeiras-Roubín S, Abu-Assi E, et al. Mehran contrast nephropathy risk score: is it still useful 10 years later? J Cardiol 2016;67:262-7.

32 Bandle B, Ward K, Min S-J, et al. Can Braden score predict outcomes for hospitalized heart failure patients? J Am Geriatr Soc 2017;65:1328-32.

33 Nakahashi T, Tada H, Sakata K, et al. Impact of concomitant peripheral artery disease on contrast-induced acute kidney injury and mortality in patients with acute coronary syndrome after percutaneous coronary intervention. Heart Vessels 2020;35:1360-7.

34 Lüders F, Malyar N, Meyborg M, et al. Impact of peripheral arterial occlusive disease on the development of contrast medium-induced acute kidney injury. Nephron Clin Pract 2012;122:38-43.

35 Clegg A, Kring D, Plemmons J, et al. North Carolina wound nurses examine heel pressure ulcers. J Wound Ostomy Continence Nurs 2009;36:635-9.

36 Park KH. A retrospective study using the pressure ulcer scale for healing (push) tool to examine factors affecting stage II pressure ulcer healing in a Korean acute care hospital. Ostomy Wound Manage 2014;60:40-51.

37 Ranzani OT, Simpson ES, Japiassú AM, et al. The challenge of predicting pressure ulcers in critically ill patients. A multicenter cohort study. Ann Am Thorac Soc 2016;13:1775-83.

38 Tierney S, Cook G, Mamas M, et al. Nurses' role in the acute management of patients with non-ST-segment elevation acute coronary syndromes: an integrative review. Eur $J$ Cardiovasc Nurs 2013;12:293-301.

39 Cohen A, Assyag P, Boyer-Chatenet L, et al. An education program for risk factor management after an acute coronary syndrome: a randomized clinical trial. JAMA Intern Med 2014;174:40-8. 